\title{
Estudo comparativo da perceção de resiliência por pais e crianças/adolescentes
}

Perception of resilience in parents and children/adolescents: a comparative study Estudio comparativo de la percepción de resiliencia por padres y niños/adolescentes

Graça Aparício io https://orcid.org/0000-0001-6149-062X:

Manuela Ferreira io https://orid.org/0000-0002-8452-2222:

João Duarte i https://oridid.org/0000-0001-7082-8012

Ernestina Silva io https://orid. org/0000-0002-4401-6296/

Madalena Cunha io https://orcid.org/0000-0003-0710-92201

Isabel Bica io hitps:///orid.org/0000-0002-7019-01321

Carlos Albuquerque io https://orcid.org/0000--0002-2297-0636'

Lídia Cabral io https://orcid.org/0000-0001-7306-50491

Como citar:

Aparício G, Ferreira M, Duarte J, Silva E, Cunha M, Bica I, et al. Estudo comparativo da perceção de resiliência por pais e crianças/adolescentes. Acta Paul Enferm. 2020;33:e-APE20190178

DOI

http://dx.doi.org/10.37689/ acta-ape/2020A00178

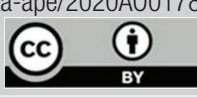

Descritores

Crianças; Adolescentes; Pais; Resiliência psicológica; Percepção

Keywords

Child; Child labor; Parents; Resilience, psychological; Perception

\section{Descriptores}

Niño; Trabajo infantil; Padres; Resiliencia psicológica; Percepción

Submetido

17 de Julho de 2019

Aceito

26 de Agosto de 2019

Autor correspondente

Graça Aparício

E-mail: gaparicio5@hotmail.com

\section{Resumo}

Objetivo: Caracterizar a auto-perceção de resiliência das crianças e adolescentes; analisar as diferenças na perceção dos pais e sua relação com algumas variáveis de contexto sociodemográfico.

Métodos: Estudo transversal realizado no âmbito do Projeto MaiSaúdeMental, numa amostra não probabilística de conveniência de 567 crianças e adolescentes, 50,6\% do sexo feminino, idade entre 9-17 anos (média= 12,40; $\mathrm{Dp}=1,59$ anos) de escolas do ensino básico da região centro de Portugal e 592 pais (média idade= 40,43 anos; $\mathrm{Dp}=2,58$ anos). Utilizou-se um questionário de caracterização sociodemográfica e a subescala Internal Assets do Healthy Kids Resilience Assessment Module (versão 6.0), adaptada à população Portuguesa por Martins (2005), composta por 18 itens e seis dimensões.

Resultados: Das crianças / adolescentes $78,8 \%$ moravam com os pais. Dos progenitores a maioria tinha entre 40 e 41 anos. A resiliência foi classificada como moderada por $47,8 \%$, das crianças / adolescentes, numa distribuição idêntica pelos pais. 0 test-t mostrou que as crianças têm uma auto-percepção mais positiva de resiliência, face à percepção dos pais, com diferenças significativas em todas as dimensões $(p<0,000)$. Os pais mais jovens têm uma perceção mais positiva da resiliência dos filhos, mas apenas significativa na empatia $(p=0,036)$ e resolução de problemas $(p=0,001)$. A resiliência diminuiu significativamente com 0 aumento da idade e escolaridade e foi mais elevada em crianças que vivem com os pais.

Conclusão: Os resultados do estudo evidenciam diferenças entre a perceção de resiliência nas crianças e pais, sendo esta influenciada por características sociodemográficas.

\section{Abstract}

Objectives: To characterize self-perception of resilience in children and adolescents, and to analyze how this self-perception differs from the perception of their parents in correlation with sociodemographic variables.

Methods: This was a cross-sectional study conducted as part of the MAISaúdeMental (More Mental Health) project with a nonprobability convenience sample including 567 children and adolescents, $50.6 \%$ of whom were females aged between 9 and 17 years old (mean $=12.40$; $S D=1.59$ years old) enrolled in basic education schools from Central Portugal, and 592 parents (mean age $=40.43$ years old; $S D=2.58$ years old). A questionnaire for sociodemographic characterization was used, along with the Healthy Kids Resilience Assessment Module (version 6.0) Internal Assets subscale, adapted to the Portuguese population by Martins (2005), composed of 18 items and 6 dimensions.

Results: Out of the total number of children/adolescents, $78.8 \%$ lived with their parents. Out of the total number of parents, most were between the ages of 40 and 41 years old. Resilience was classified as moderate by $47.8 \%$ of children/adolescents at an identical distribution in parents. The t-test showed children's self- 
perception of resilience to be more positive when compared to their parents with significant differences seen in all dimensions $(p<0.000)$. Younger parents showed a more positive perception of their children's resilience, significant only for "empathy and respect" (0.036) and "problem-solving skills" (0.001). Resilience decreased significantly with age and higher education levels, and children living with their parents showed higher resilience.

Conclusion: Study results show differences between the perceptions of resilience in children and their parents, which are influenced by sociodemographic characteristics.

\section{Resumen}

Objetivo: Caracterizar la autopercepción de resiliencia de los niños y adolescentes; analizar las diferencias en la percepción de los padres y su relación con algunas variables de contexto sociodemográfico.

Métodos: Estudio transversal realizado en el ámbito del Proyecto "MaiSaúdeMental" (Más Salud Mental), en un muestreo no probabilístico de conveniencia de 567 niños y adolescentes, 50,6\% de sexo femenino, edad entre 9 y 17 años (promedio=12,40; Dp=1,59 años) de escuelas primarias de la región centro de Portugal y 592 padres (edad promedio= 40,43 años; $\mathrm{Dp}=2,58$ años). Se utilizó un cuestionario de caracterización sociodemográfica y la subescala Internal Assets del Healthy Kids Resilience Assessment Module (versión 6.0), adaptada a la población portuguesa por Martins (2005), compuesta por 18 ítems y 6 dimensiones.

Resultados: De los niños/adolescentes, 78,8\% vivían con los padres. De los progenitores, la mayoría tenía entre 40 y 41 años. La resiliencia fue clasificada como moderada por el 47,8\% de los niños/adolescentes, en una distribución idéntica por los padres. El test-T mostró que los niños tienen una autopercepción más positiva de resiliencia, frente a la percepción de los padres, con diferencias significativas en todas las dimensiones $(p<0,000)$. Los padres más jóvenes tienen una percepción más positiva de la resiliencia de los hijos, pero poco significativa en la empatía $(p=0,036)$ y resolución de problemas $(p=0,001)$. La resiliencia se redujo significativamente con el aumento de la edad y escolaridad y fue más elevada en niños que viven con los padres.

Conclusión: Los resultados del estudio dejan en evidencia diferencias entre la percepción de resiliencia en los niños y padres, de modo que está influenciada por características sociodemográficas.

\section{Introdução}

Analisar a adaptação às adversidades da vida tem sido tema recorrente de investigação, dada a sua importância na identificação de recursos a mobilizar para promover resultados positivos em ambientes adversos, nomeadamente na prevenção de comportamentos de risco e na promoção da saúde mental na infância e adolescência Alguns autores ${ }^{(1-3)}$ definem resiliência como um processo interactivo e dinâmico, que caracteriza uma capacidade particular do indivíduo para resistir ou se adaptar às adversidades da vida. Mais tarde Rutter ${ }^{(4)}$ reconheceu que existe uma enorme heterogeneidade nas respostas aos diversos tipos de adversidades ambientais, face às evidências de que alguns indivíduos têm um resultado melhor do que outros perante um nível comparável de adversidade, ressalvando a importância da mediação ambiental do risco e dos vários fatores que contribuem para o aumento da vulnerabilidade e/ou adaptação às adversidades, nomeadamente a interação entre as características de natureza individual e o ambiente. ${ }^{(4)}$ De facto as pesquisas iniciais sobre resiliência dirigiam-se sobretudo a crianças em ambientes de alto risco, nomeadamente familiar e social, considerando a origem da resiliência enquanto característica da criança sujeita a adversidades, tal como viver em pobreza extrema ou sob abuso recorrente e exposta a stresse excessivo ou prolongado. Investigações posteriores foram reconhecendo cada vez mais a família e os fatores sociais como os elementos que podem auxiliar a criança a compensar as várias influências do contexto ambiental. ${ }^{(5)} \mathrm{O}$ apoio e relacionamentos de qualidade com adultos, podem constituir uma oportunidade para a construção de habilidades de adaptação à adversidade. $^{(6,7)}$

Nesta conjuntura existe atualmente uma visão mais positiva e integradora de resiliência, associando o seu conceito a modelos baseados nas forças individuais e focado na compreensão dos processos epigenéticos e neurobiológicos do cérebro em desenvolvimento, com enfoque particular nos pontos de viragem e seu efeito nas experiências que aumentam as oportunidades e a capacidade de enfrentamento perante as adversidades. ${ }^{(8,9)}$ Estas investigaçôes revestem-se de grande importância, sobretudo pelos benefícios que aportam na perspectiva das crianças e jovens.

Os recursos de resiliência na criança / jovem, incluem diferentes domínios, nomeadamente recursos internos, ambiente familiar e ambiente social. As características internas, recurso em foco neste estudo, representam as forças e competências individuais, como temperamento, comunicação, capacidades de aprendizagem e de auto-eficácia, 
auto-estima e habilidades adaptativas, construtos considerados protetores, que se desenvolvem cedo na vida de forma dinâmica e progressiva, mas sujeitos às variaçóes contextuais e desenvolvimentais, como o género e idade. Nesta perspetiva, algumas pesquisas $^{(1,10)}$ verificaram que o sexo feminino manifesta habitualmente uma resiliência mais positiva que o sexo oposto e que esta vai decrescendo com a idade. ${ }^{(11)}$ Desta forma, as circunstâncias da vida têm um impacto real na promoção da resiliência. Locais como a escola, a casa e o centro de saúde, podem ser potenciais promotores de resiliência, detendo os enfermeiros um papel privilegiado nessa promoção, sobretudo na sua intervenção com a criança/família em âmbito comunitário. Neste domínio e através da mobilização dos recursos de saúde e/ou da comunidade, os enfermeiros detêm um papel central na co-criação de oportunidades que permitam à criança/família o desenvolvimento de competências para a identificação e mobilização dos factores protectores de que dispóem e criando condiçóes para que construam uma trajectória de vida saudável. ${ }^{(12)}$ Porém, intervenções promotoras de resiliência não deverão surgir isoladamente, mas sim integradas na vida quotidiana da criança/família. Outras investigaçóes $^{(13,14)}$ que analisaram a resiliência familiar e do ambiente escolar, permitiram verificar que por um lado as opinióes dos pais sobre a resiliência de seus filhos se relacionam com as suas percepçôes sobre as habilidades e o sucesso escolar da criança. Por outro lado, as crianças atribuem diferentes significados ao fenómeno, associando-o a expectativas da família, ao facto de lhes agradar a si e aos outros, o que os leva a adaptar-se a essa categorização. Ou seja, as opinióes dos alunos sobre a sua própria resiliência parecem depender da sua perceção da visão dos professores e pais.

Evidências têm salientado a importância dos primeiros anos de vida na construção dos alicerces para um desenvolvimento psicossocial saudável. ${ }^{15-}$ 17) As relaçôes estáveis de vinculação e apego com os pais e outros cuidadores, criam as bases para o funcionamento fisiológico das crianças, para as interpretaçóes emocionais e cognitivas das suas experiências sociais e para a aquisição de significados sobre si mesma e sobre os outros em diversas situaçôes sociais, tendo um impacto importante na sua reatividade ao stresse, no seu desempenho social e para a saúde física e mental das crianças. Se uma criança recebe cuidado afetuoso quando necessita e apoio à autonomia durante as suas experiências de exploração do ambiente, ela desenvolve um senso de valor de si própria e uma crença na disponibilidade dos outros. Este sentimento de apego seguro é considerado um fator de proteção individual importante, tendo sido associado a melhores resultados posteriores em áreas como autoconfiança, auto-eficácia, empatia e competência social em crianças em idade escolar e na adolescência. Numa outra perspectiva, esta relação diádica que se estabelece entre as crianças e os seus cuidadores, tende a promover de igual forma sentimentos de eficácia nos cuidadores, em resultado da perceção de competência em interpretar e responder adequadamente às diversas solicitaçôes da criança. Os estudos indicam ainda que essas forças relacionais, se positivas, têm o potencial de compensar experiências de parentalidade abusiva ou estressante. ${ }^{(18,19)}$ Ainda neste âmbito, investigaçôes documentam que as cogniçóes parentais sobre o desenvolvimento/competências dos filhos influenciam a forma como os pais se relacionam com eles e esses comportamentos tendem a influenciar o desenvolvimento infantil, num processo de retro feedback, afirmando-se que cogniçóes parentais positivas, contribuem para uma melhor adequação às necessidades desenvolvimentais dos filhos. ${ }^{(20,21)}$ No âmbito da resiliência, a perspetiva parental está relacionada com a perceção que os pais têm das habilidades dos seus filhos, podendo também associar-se à sua posição social e género. Num estudo de Zolkoski e Bullock ${ }^{(22)}$ foram os pais com menos idade que percecionaram de forma mais positiva a resiliência dos filhos, quase em todas as dimensóes dos recursos internos, com destaque para a autoconsciência, cooperação e comunicação e resolução de problemas. Outro estudo realizado com 393 estudantes do ensino secundário, com idades entre os 14 e os 21 anos de três escolas do norte de Portugal mostrou que a resiliência era influenciada pelo ambiente familiar, escolar e a relação com os pares, revelando-se o ambiente familiar como o principal preditor de resiliência. ${ }^{(1)} \mathrm{O}$ mesmo estudo apurou que a resi- 
liência se associou com o sexo feminino e com a idade, indicando que os estudantes mais novos tinham melhores níveis de resiliência. ${ }^{(1)}$

Neste enquadramento, este estudo tem como objetivos caracterizar a auto-perceção de resiliência das crianças e adolescentes, analisar as diferenças na perceção dos pais e a sua relação com algumas variáveis de contexto sociodemográfico.

\section{Métodos}

Estudo quantitativo, transversal e descritivo, realizado no âmbito de um projecto de promoção da saúde mental em crianças e adolescentes em contexto escolar, designado MaiSaúdeMental, referência CENTRO-01-0145-FEDER-023293, em curso na Escola Superior de Saúde do Instituto Politécnico de Viseu, Portugal. A amostra era constituída por 567 crianças / adolescentes com idade entre 9 e 17 anos (média $=12,40, \mathrm{DP}=1,591$ ) de um Agrupamento de Escolas básicas / secundárias da região centro de Portugal, e seus pais (pai e mãe), num total de 592 (média idade $=40,43$ anos; $\mathrm{Dp}=2,58$ anos), selecionados de forma não probabilística por conveniência.

Utilizou-se um questionário de caracterização sociodemográfica e para avaliação e compreensão dos fatores internos de resiliência associados ao desenvolvimento saudável, foi aplicada a versão crianças e adolescentes e versão para pais, do questionário "Healthy Kids Resilience Assessment Module" (Versão 6.0), adaptada à população Portuguesa por Martins. (23) A escala original inclui as sub-escalas external e internal assets e o Response-set Breakers. A subescala Internal Assets, utilizada neste estudo, integra os resultados desenvolvimentais positivos ou traços de resiliência e compreende 18 itens com as seguintes seis dimensóes: Cooperação e Comunicação; Autoeficácia; Empatia; Resolução de Problemas; Autoconsciência; Objetivos e Aspiraçôes. São classificados numa Escala tipo Likert com quatro possibilidades de resposta, em que: $1=$ Nem tudo é Verdadeiro, e 4= Muito Verdadeiro e os scores são obtidos pela soma total dos itens, divididos pelo número de itens de cada subescala. Neste estudo os scores obtidos em cada dimensão foram convertidos numa escala de 0 a 100, correspondendo respetivamente aos resultados mínimos e máximos obtidos em cada dimensão. $\mathrm{O}$ nível de resiliência foi classificado numa escala de percentis, sendo os pontos de corte obtidos, abaixo, entre e acima dos percentis 25 e 75, considerados como baixo, moderado e alto nível de resiliência.

Os requisitos éticos foram salvaguardados, através da solicitação de parecer à Comissão Nacional de Proteção de Dados (Refa 03.01, 38790 de 18/12/2017) e posteriormente foi efetuada a submissão do protocolo de pesquisa à Direção Geral de Educação (DGE), (inquérito n. ${ }^{\circ} 0012100017$ ). Após aprovação do estudo, foi solicitada permissão ao Conselho Diretivo do Agrupamento Escolar parceiro e obtido o consentimento livre e informado dos encarregados de educação, seguindo-se a seguinte dinâmica para a recolha de informação: Os questionários dos pais foram entregues a cada criança/ adolescente pelos diretores de turma em envelope fechado juntamente com o consentimento informado para autorização da participação dos filhos; os pais que aceitaram integrar o estudo preencheram o questionário que foi devolvido em envelope fechado ao diretor de turma, assim como o consentimento assinado. As crianças/adolescentes cujos pais autorizaram a participação foram preencheram os questionários em sala de aula e estes foram posteriormente entregues pelo director de turma aos investigadores responsáveis.

O tratamento estatístico foi realizado utilizando o Statistical Package for the Social Sciences ${ }^{\circledR}$ (SPSS - versão 24.0), com base na análise estatística descritiva e analítica, com recurso à análise de variância ANOVA e ao Teste-t para diferença de médias, utilizando-se o Intervalo de Confiança (IC) a 95\% (significância $\mathrm{p}<0,05$ ).

\section{Resultados}

\section{Caracterização sociodemográfica das crianças / adolescentes e pais}

Da amostra de crianças/adolescentes, $38.4 \%$ pertenciam ao grupo etário dos 12-13 anos (média idade= 12.41; Dp=1.615), seguidos dos abaixo dos 11 anos 
(33.5\%), em percentagens bastante idênticas entre géneros. Relativamente ao contexto familiar, $77.4 \%$ viviam com os pais, enquanto $18.5 \%$ viviam apenas com a mãe, $82.2 \%$ com um irmão e $17.8 \%$ com dois ou mais irmáos. $\mathrm{Na}$ escola a maioria frequentava o $9^{\circ}$ e $8^{\circ}$ ano de escolaridade $(22.8 \%$ e $21.5 \%$ respetivamente), seguidos dos que frequentava o $6^{\circ}, 7^{\circ}$ e $5^{\circ}$ anos, respetivamente $21.0 \%, 17.8 \%$ e $16.9 \%$, sem diferenças significativas entre os géneros. Os progenitores tinham uma média de idade de 40,43 anos $(\mathrm{D} p=2,58)$, a maioria pertencia ao grupo etário dos 40-41 anos (38.3\%) seguidos daqueles com menos de 39 anos $(32,9 \%)$ e 84,8\% eram mães.

\section{Caracterização do nível de Resiliência}

Os valores médios da sub-escala situaram-se entre $88.32(\mathrm{Dp}=16.25)$ na dimensão objetivos e aspiraçóes e $70.48(\mathrm{Dp}=19.73)$ na autoeficácia. Estes resultados indicam que os recursos internos, ou seja, os traços de resiliência das crianças / adolescentes do estudo, podem ser considerados altos, sendo que as dimensões que mais contribuíram para uma perceção de resiliência mais positiva foram os objetivos e aspirações e autoconsciência, face aos seus valores médios. Analisando por género (Tabela 1), apuramos que as meninas apresentam médias mais elevadas que os rapazes em todas as dimensóes, exceto na autoeficácia e autoconsciência, mas apenas significativas na cooperação e comunicação $(t=-2$. 194; $\mathrm{p}=0.029)$ e empatia $(\mathrm{t}=4.637 ; \mathrm{p}=0.000)$. Face à idade, a análise de variância ANOVA indicou valores médios mais elevados e significativos em todas as dimensóes da escala $(\mathrm{p}<0,05)$ nas crianças abaixo dos 11 anos, exceto na dimensão objetivos e aspiraçôes $(\mathrm{F}=1.857 ; \mathrm{p}=0.157)$ e uma mesma tendência relativamente ao nível de escolaridade.

Considerando os pontos de corte definidos na metodologia, a classificação de resiliência das crianças/adolescentes foi para a maioria considerada como moderada (47.8\%), alta em 31.2\% e baixa em $21.0 \%$ numa tendência similar entre os géneros e dessa forma sem significância estatística (Qui-quadrado $=4.797 ; \mathrm{p}=0,091$ ). $\mathrm{Na}$ perspectiva dos pais e analisando a tabela 3., constata-se que a média mais elevada corresponde à dimensão objetivos e aspiraçóes $(M=77,06 \pm 21.75)$ seguida da Resolução de problemas $(\mathrm{M}=72,11 \pm 21.05)$ e da Empatia $(M=71,55 \pm 18,07)$. Os coeficientes de variação indiciam uma dispersão moderada face às médias. No que respeita à classificação e para o global da amostra, 48,0\% consideram as crianças/adolescentes como moderadamente resilientes e 27,0\% e $25,0 \%$ muito e pouco resilientes respetivamente (Tabela 2). Esta tendência verifica-se na análise da resiliência por parte da mãe e do pai, contudo uma maior percentagem de mães considera o filho moderadamente resiliente $(48,4 \%)$ enquanto mais pais $(28,9 \%)$ os consideram muito resilientes, porém sem significância estatística (Qui-quadrado= 0,279; $\mathrm{p}=0,870)$.

Tabela 1. Estatísticas das seis dimensões e total da sub-escala de resiliência, em função do género das crianças/adolescentes

\begin{tabular}{|c|c|c|c|c|c|c|c|c|}
\hline \multirow{2}{*}{ Dimensões } & \multirow{2}{*}{ Média } & \multirow{2}{*}{$\mathrm{DP}^{*}$} & \multicolumn{2}{|c|}{ Meninas } & \multicolumn{2}{|c|}{ Rapazes } & \multicolumn{2}{|c|}{ T-test } \\
\hline & & & Média & $\mathrm{DP}^{*}$ & Média & $\mathrm{DP}^{\star}$ & $\mathrm{t}$ & p-value+ \\
\hline Cooperação e Comunicação & 75.60 & 18.85 & 77.31 & 18.29 & 73.84 & 19.28 & $-2,194$ & 0,029 \\
\hline Autoeficácia & 70.48 & 19.73 & 69.06 & 19.85 & 71.94 & 19.55 & 1,739 & 0,083 \\
\hline Empatia & 74.79 & 20.24 & 78.63 & 18.86 & 70.87 & 20.89 & $-4,637$ & 0,000 \\
\hline Resolução problemas & 75.26 & 22.23 & 75.61 & 23.22 & 74.92 & 21.22 & $-0,369$ & 0,713 \\
\hline Autoconsciência & 78.95 & 18.77 & 77.47 & 19.51 & 80.48 & 17.89 & 1,912 & 0,056 \\
\hline Objetivos e aspirações & 88.32 & 16.25 & 88.97 & 15.44 & 87.66 & 17.06 & $-0,958$ & 0,339 \\
\hline Resiliência total & 77.23 & 14.23 & 77.84 & 14.09 & 76.62 & 14.38 & $-1,022$ & 0,307 \\
\hline
\end{tabular}

*DP - Desvio-Padrão; +T-test

Tabela 2. Perceção dos pais da resiliência das crianças e adolescentes em função do género

\begin{tabular}{|c|c|c|c|c|c|c|c|c|c|c|}
\hline \multirow{2}{*}{$\begin{array}{l}\text { Sexo } \\
\text { Resiliência }\end{array}$} & \multicolumn{2}{|c|}{ Masculino } & \multicolumn{2}{|c|}{ Feminino } & \multicolumn{2}{|c|}{ Total } & \multicolumn{2}{|c|}{ Residuais } & \multirow{2}{*}{$\mathrm{X}^{2}$} & \multirow{2}{*}{$p$-value+ } \\
\hline & $n(90)$ & $\%(15,2)$ & $n(502)$ & $\%(84.8)$ & $\mathrm{n}(592)$ & $\%(100.0)$ & 1 & 2 & & \\
\hline Pouca & 23 & 25,6 & 125 & 24,9 & 148 & 25 & 0,1 & $-0,1$ & 0,279 & 0,870 \\
\hline Moderada & 41 & 45,6 & 243 & 48,4 & 284 & 48 & $-0,5$ & 0,5 & & \\
\hline Elevada & 26 & 28,9 & 134 & 26,7 & 160 & 27 & 0,4 & $-0,4$ & & \\
\hline Total & 90 & 100,0 & 502 & 100,0 & 592 & 100.0 & & & & \\
\hline
\end{tabular}

(\%) - percentual; +Qui-Quadrado 
Considerando que a idade dos pais pode influenciar a sua perceção, foi efetuada esta análise, verificando-se pelo Test-t para diferença de médias, serem os pais mais novos os que percecionam mais positivamente a resiliência dos seus filhos, com valores médios mais elevados em todas as dimensóes, mas apenas com significância estatística na empatia $(\mathrm{p}=0,036)$, na resolução de problemas $(\mathrm{p}=0,001)$ e na resiliência total $(p=0,047)$. Tal como apresentado na tabela 3, para analisarmos a diferença entre a perceção da resiliência dos pais e das crianças/jovens foi realizado o Teste-t para diferença de médias, constatando-se que as crianças apresentam valores médios mais elevados e significativos em todas as dimensões dos recursos internos de resiliência, quando comparadas com os pais $(\mathrm{p}<0,05)$. Os objetivos e aspiraçôes, autoconsciência, cooperação e comunicação e resolução de problemas foram os recursos internos que mais contribuíram na perceção alta de resiliência por parte das crianças/adolescentes, enquanto nos pais se evidencia a sua perceção dos construtos objetivos e aspiraçóes, resolução de problemas e empatia.

Tabela 3. Estatísticas das seis dimensões e total da sub-escala de resiliência, em função dos grupos de crianças / adolescentes e pais

\begin{tabular}{|c|c|c|c|c|c|c|}
\hline \multirow{2}{*}{$\begin{array}{l}\text { Percepção por } \\
\text { grupos } \\
\text { Dimensões }\end{array}$} & \multicolumn{2}{|c|}{$\begin{array}{c}\text { Crianças/ } \\
\text { adolescentes }\end{array}$} & \multicolumn{2}{|c|}{ Pais } & \multicolumn{2}{|c|}{ T-test } \\
\hline & Média & $D p^{\star}$ & Média & $\mathrm{DP}^{*}$ & $\mathrm{t}$ & $p$-value+ \\
\hline $\begin{array}{l}\text { Cooperação e } \\
\text { Comunicação }\end{array}$ & 75.60 & 18.85 & 69.85 & 18.80 & 5.193 & 0.000 \\
\hline Autoeficácia & 70.48 & 19.73 & 62.36 & 19.13 & 7.111 & 0.000 \\
\hline Empatia & 74.79 & 20.24 & 71.54 & 18.07 & 2.881 & 0.004 \\
\hline $\begin{array}{l}\text { Resolução } \\
\text { problemas }\end{array}$ & 75.26 & 22.23 & 72.10 & 21.05 & 2.485 & 0.013 \\
\hline Autoconsciência & 78.95 & 18.77 & 68.93 & 20.61 & 8.637 & 0.000 \\
\hline $\begin{array}{l}\text { Objetivos e } \\
\text { aspirações }\end{array}$ & 88.32 & 16.25 & 77.06 & 21.74 & 9.948 & 0.000 \\
\hline Resiliência total & 77.23 & 14.23 & 70.31 & 15.66 & 7.867 & 0.000 \\
\hline
\end{tabular}

\section{Discussão}

Atuar no ambiente infanto-juvenil, particularmente no contexto escolar, frequentemente adverso à saúde mental, é um desafio emergente face aos novos paradigmas da saúde e em particular da saúde escolar. Este estudo permitiu identificar um perfil das forças e recursos internos desta amostra de crianças/adolescentes, bem como das perceçóes daque- les do seu contexto mais próximo, os pais, de forma a dar resposta a um dos desígnios do projeto MaiSaúdeMental.

Os resultados indicaram que as crianças e adolescentes tinham no geral uma auto-perceção positiva da sua resiliência, salientando-se os domínios objetivos e aspiraçôes e autoconsciência, recursos internos importantes para enfrentamento das adversidades, ou na visão de alguns autores, ${ }^{(3,4)}$ factores protectores e promotores de resiliência, dado que estão associados, sobretudo a autoconsciência, à compreensão das próprias emoções, forças, fraquezas e necessidades. Estes recursos transportam-nos para a visão mais positiva e integradora da resiliência, ${ }^{(8,9)}$ associada a modelos baseados nas forças internas individuais e que vão sendo desenvolvidos através da exposição natural e gradual a dificuldades em níveis administráveis. Além disso enquadra-se na perspectiva de que a resiliência enquanto processo não é imutável, antes desenvolve-se e modifica-se ao longo da vida, necessitando do equilíbrio entre factores de risco e proteção e de capacidade de os gerir, de acordo com as características desenvolvimentais. Este facto parece justificar também as diferenças associadas a factores individuais, como o género e idade. Neste estudo apurou-se, tal como em diversas pesquisas nacionais e internacionais ${ }^{(1,11,13)}$ que as meninas apresentavam uma perceção dos seus recursos internos mais positiva face ao sexo oposto, exceto na autoeficácia e autoconsciência e que esta vai diminuindo com o aumento da idade. A diferenciação face ao género será explicada pela maturação desenvolvimental diferente do sexo feminino que lhe permite desenvolver competências pró-socias adaptativas mais precocemente, tais como a empatia e a cooperação e comunicação. Contudo, a transição para a adolescência acarreta igualmente alguma vulnerabilidade e desorganização pessoal, familiar e na relação com o grupo de pares, o que estará na base da auto-perceção menos positiva de resiliência, associada ao aumento da idade. Contudo, as evidências $^{(2,6,21,22)}$ salientam que o apoio e relacionamentos de qualidade com adultos podem constituir uma oportunidade para a construção de habilidades de adaptação, salientando-se a influência do ambiente familiar próximo, nomeadamente dos 
pais e as suas cogniçóes sobre o desenvolvimento/ competências dos filhos. Neste âmbito, as habilidades dos pais em monitorar e identificar comportamentos sociais adequados e inadequados dos filhos é um primeiro passo para que as práticas parentais educativas sejam mais ajustadas e melhor sucedidas. Além disso, os estudos documentam que a perceção dos pais sobre a resiliência dos filhos tende a associar-se à sua perceção sobre as competências e sucesso escolar das crianças, o que estará fortemente ligado às suas aspiraçóes e expectativas para o sucesso académico dos filhos ${ }^{(14)}$. Porém, no atual estudo constatou-se que as crianças apresentavam uma auto-perceção de resiliência mais positiva, quando comparada com a dos seus pais, o que, face às evidências, pode ser indicador de algum comprometimento nas relaçóes de confiança existentes nestes relacionamentos e consequentemente na adequação das responsabilidades parentais às necessidades dos filhos. Apurou-se ainda serem os pais mais novos os que percecionam mais positivamente a resiliência dos seus filhos, tal como outros estudos documentam. $^{(22,23)}$ Estes resultados permitiram identificar um foco de atenção para a intervenção dos enfermeiros no âmbito do projecto MaiSaúdeMental, e para a definição de estratégias para a promoção de resiliência na criança/família no contexto escolar e comunitário, mobilizando os recursos de saúde e/ou da comunidade para uma vida mais saudável. ${ }^{(12)}$

Porém, estas evidências têm que ser analisadas face a algumas limitaçóes, quer resultantes do número limitado de crianças estudadas, das características não aleatórias da amostra, como do caráter transversal do estudo. Estes factos, que limitam a generalização dos resultados, podem então ser entendidos como um "retrato" da vivência de um constructo dinâmico e uma análise estática de perceçôes que se reconhece, se vão alterando à medida que a criança cresce e se desenvolve. Apesar disso, identificar e compreender as características do grupo alvo da nossa intervenção, facilitou a definição das estratégias a implementar para o fortalecimento dos recursos adaptativos e de sucesso deste grupo, permitindo intervir na conjuntura social atual em que as adversidades no contexto familiar e ambiental, nomeadamente na escola, são frequentes.

\section{Conclusão}

Os resultados deste estudo permitem traçar um perfil das forças internas destas crianças e adolescentes, caracterizado maioritariamente por uma auto-perceção de resiliência moderada, contribuindo de forma mais expressiva os recursos internos objetivos e aspirações, autoconsciência, cooperação, comunicação e a resolução de problemas. As meninas e as crianças mais novas (menos 11 anos) classificam os seus recursos internos de resiliência de forma mais positiva. Os pais têm, na sua maioria, uma perceção moderada da resiliência dos filhos, salientando-se os recursos internos objetivos e aspiraçóes, resolução de problemas e empatia, sendo os pais mais novos o que têm uma perceção mais positiva. Verificouse diferença estatística significante entre a perceção de resiliência das crianças/adolescentes e a dos seus pais, apresentando as crianças valores mais elevados em todas as dimensóes dos recursos internos.

\section{Agradecimentos}

O nosso agradecimento ao Fundo Social Europeu, ao Programa Operacional CENTRO 2020 e à Fundação para a Ciência e Tecnologia do Governo Português, instituiçóes públicas que se constituem como fontes de financiamento do Projeto de Investigação MAISaúdeMental (código de identificação:CENTRO-01-0145-FEDER-023293), onde este estudo se integra.

\section{Colaborações}

Aparício G, Ferreira M, Duarte J, Silva E, Cunha M, Bica I, Albuquerque C e Cabral L contribuíram com a concepção do estudo, análise e interpretação de dados, elaboração do artigo,revisão crítica do conteúdo e aprovação final da versão a ser publicada.

\section{Referências}

1. Dias PC, Cadime I. Protective factors and resilience in adolescents: The mediating role of self-regulation. Psicol Educ. 2017;23(1):3743. 
2. UNICEF. Actions for children and youth resilience: Guide for governments [Internet]. Panama: UNICEF; 2013 [cited 2019 Jan 11]. Available from: http://repo.floodalliance.net/sspui/ bitstream/44111/1209/1/Actions\%20for\%20Children\%20and\%20 Youth\%20ResilienceGuide\%20for\%20Governments.pdf

3. Rutter M. Resilience, competence, and coping. Child Abuse Negl. 2007;31(3):205-9.

4. Rutter M. Resilience as a dynamic concept. Dev Psychopathol. 2012; 24(2):335-44.

5. Sameroff, A. Resiliência precoce e suas consequências para 0 desenvolvimento. In: Tremblay RE, Boivin M, Peters RD, Masten AS, editores. Enciclopédia sobre o Desenvolvimento na Primeira Infância [Internet]. Centro de Excelência para o Desenvolvimento na Primeira Infância; 2011 [citado 2019 Jan 8]. Disponível em: http://www.enciclopedia-crianca.com/ sites/default/files/dossiers-complets/pt-pt/resiliencia.pdf

6. Center on the Developing Child. Supportive relationships and active skill-building strengthen the foundations of resilience: Working Paper No. 13. Cambridge, USA: Harvard University; 2015

7. Habilidades parentais. In: Tremblay RE, Boivin M, Peters RD, editors. Enciclopédia sobre o desenvolvimento na primeira infância [Internet]. Centro de Excelência para o Desenvolvimento na Primeira Infância; 2011 [citado 2019 Jan 1]. Disponível em: http://www.enciclopedia-crianca.com/ sites/default/files/dossiers-complets/pt-pt/habilidades-parentais.pdf

8. Benson PL, Scales PC, Hamilton SF, Sesma Jr A, Hong KL, Roehlkepartain EC. Positive youth development so far: Core hypotheses and their implications for policy and practice. Insights \& Evidence. 2006 ;3(1):1-13.

9. Wright M, Masten A, Narayan A. Resilience processes in development: Four waves of research on positive adaptation in the context of adversity. In: Goldstein S, Brooks R, editors. Handbook of resilience in children [Internet]. New York: Springer; 2013.

10. Bernardo SC, editor. Auto-percepções da capacidade de resiliência em jovens afro portugueses residentes num bairro desfavorecido. Portugal: Universidade do Minho; 2009.

11. Sun J, Stewart D. Age and gender effects on resilience in children and adolescents. Int J Mental Health Promot. 2012; 9(4):16-25.

12. Botas AF. Promover a resiliência na criança/família: um percurso de desenvolvimento de competências (tese). Portugal: Universidade Católica Portuguesa; 2011.
13. Löfgren H, Löfgren R. Grades in the eyes of our parents: a narrative approach to educational resilience in pupils' stories of getting their first grades. Nordic J Stud Educ Pol. 2017;3(2):165-78.

14. Kärkkäinen R, Räty H, Kasanen K. Do mothers and fathers perceive their child's academic potential in a similar way? Educ Stud. 2016. 42(5):533-6.

15. Grossmann K, Grossmann KE. 0 impacto do apego à mãe e ao pai e do apoio sensível à exploração nos primeiros anos de vida sobre 0 desenvolvimento psicossocial das crianças até o início da vida adulta. In: Tremblay RE, Boivin M, Peters RD, editors. Enciclopédia sobre 0 desenvolvimento na primeira infância [Internet]. Centro de Excelência para o Desenvolvimento na Primeira Infância; 2011. [citado 2019 Jan 8]. Disponível em: http://www.enciclopedia-crianca.com/sites/default/ files/dossiers-complets/pt-pt/apego.pdf

16. Fox NA, Hane AA. Studying the biology of human attachment. In: Cassidy J, Shaver PR, editores. Handbook of attachment: Theory, research, and clinical applications. 2nd ed. New York: Guilford Press; 2008.

17. Tamura T. Effect of perceived parent-child relationship in childhood on resilience in Japanese youth. Pschol Rep. 2018 https://doi. org/10.1177/0033294118762504.

18. Hess CR, Teti, DM, Hussey-Gardner B. Self-efficacy and parenting of high-risk infants: The moderating role of parent knowledge of infant development. J Appl Dev Psychol. 2004;25(4):423-37.

19. De la Rosa IA, Perry J, Johnson V. Pathways to resilience: Enhancing family well-being with a home visitation model. J Youth Dev. 2015; 10(1):1-26.

20. Ribas RC, Moura ML, Bornstein MH. Cognições maternas acerca da maternidade e do desenvolvimento humano: uma contribuição ao estudo da psicologia parental. Rev Bras Cresc Desenvolv Hum. 2007;17(1):104-13.

21. Sena SS, Souza LK. Percepção dos pais sobre amizade em crianças típicas e com TDAH1. Psicol_Clin. 2013; 25(1):53-72.

22. Zolkoski SM, Bullock LM. Resilience in children and youth: A review. Child Youth Serv Rev. 2012;34(12):2295-03.

23. Martins MH. Contribuições para a análise de crianças e jovens em risco/resiliência e Desenvolvimento (tese). Faro: Universidade do Algarve; 2005. 